\title{
The effect of intestinal flora on immune checkpoint inhibitors in tumor treatment: a narrative review
}

\author{
Dan Wang, He Hao, Xing Li, Zhiyu Wang \\ Department of Immuno-oncology, The Fourth Hospital of Hebei Medical University, Shijiazhuang, China \\ Contributions: (I) Conception and design: Z Wang; (II) Administrative support: Z Wang; (III) Provision of study materials: D Wang, H Hao, X Li; (IV) \\ Collection and assembly of data: D Wang, H Hao, X Li; (V) Data analysis and interpretation: All authors; (VI) Manuscript writing: All authors; (VII) \\ Final approval of manuscript: All authors. \\ Correspondence to: Zhiyu Wang, MD, PhD. Department of Immuno-Oncology, The Fourth Hospital of Hebei Medical University, Shijiazhuang, \\ China. Email: drwangzhiyu@hotmail.com.
}

\begin{abstract}
Tremendous progress has been achieved in understanding of the interaction between tumor microenvironment and intestinal flora in the past decades. Immune checkpoint inhibitors (ICIs) are a promising treatment strategy for advanced tumors, most prominently cytotoxic T-lymphocyte-associated protein (CTLA-4) and programmed cell death protein-1 (PD-1), its major ligand PD-L1, its beneficial to part of the population and obtaining excellent clinical results. However, the majority of patients do not respond or develop early progressive disease. Reached consensus by experts currently believe that the intestinal flora plays an important role in the explanation of the limited therapeutic effect of ICIs, there are differences in the composition of intestinal flora between patients with good response and patients with poor response, cloned mice by fecal microbiota transplantation (FMT) proved that the mice with transplanted feces from patients with good response can reduce tumor volume and obtain a better progress free survival (PFS). Therefore, "beneficial bacteria" seem to be enriched in the intestinal flora of patients who are wellresponsive to ICIs and can be potentially used as a marker and cancer immunotherapeutic adjuvant of ICIs. In this review, we aim to summarize some of the studies demonstrating intestinal flora on tumor immunotherapy through anti-PD1, anti-PD-L1, anti-CTLA-4 and discuss possible mechanisms of this effect.
\end{abstract}

Keywords: Intestinal flora; immune checkpoint inhibitor (ICI); tumor

Submitted Apr 29, 2020. Accepted for publication Jul 23, 2020.

doi: $10.21037 / \mathrm{atm}-20-4535$

View this article at: http://dx.doi.org/10.21037/atm-20-4535

\section{Introduction}

Immunotherapy holds much promise as a treatment for various cancers. In recent years, immune checkpoint inhibitors (ICIs) have been used to treat multiple types of tumor. Monoclonal antibodies, such as those that target the PD-1/PD-L1 or CTLA-4 checkpoints, have proved effective in treating some patients; however, for many others, the response has been poor. As the bank of clinical and basic research into ICI-based therapy has grown, a relationship between the intestinal flora and the clinical tumor immune response to ICI-based therapy has been unearthed. Based on intestinal flora sequencing, many prospective clinical studies have shown that patients with good response to ICIs treatment always have specific enrichment in the intestinal flora and that the characteristics of the intestinal flora of the patients that show good or no response are significantly different. However, the interaction between the intestinal flora and ICIs is still unclear. Different studies have even observed the same bacteria to perform different roles.

Therefore, carrying out targeted intervention based on the species of intestinal flora may become one of the methods regulating the efficacy of immunotherapy and further expanding the beneficiary population. 
We present the following article in accordance with the Narrative Review reporting checklist (available at http:// dx.doi.org/10.21037/atm-20-4535).

\section{Methods}

PubMed and Web of Science were searched for the relevant literature from the 20 years preceding July 1, 2020. The selected language is English. All literatures are published. Important references of selected articles were also reviewed. The data available in the literature were extracted and analyzed.

\section{Immune checkpoints and tumors}

Currently, PD-1/PD-L1 and CTLA-4 are the most studied checkpoints. However, there are many other immune checkpoints, such as TIM-3, IDO, OX40/OX40L, CD27/ CD70, ICOS/ICOS-L, and LAG-3. PD-1 is expressed on the surface of various immune cells such as dendritic cells (DC), natural killer (NK) cells, monocytes, regulatory $\mathrm{T}$ cells, B cells, and antigen-presenting cells (APC). PD-1 has two ligands, PD-L1 and PD-L2. PD-L1 is expressed on a variety of tumor cells, APC, and non-lymphoid organs, whereas PD-L2 is mainly expressed on immune cells such as DC and monocytes. PD-L1 is known to play a principal role in tumor immune escape.

Under normal physiological conditions, PD-1 is lowly expressed on $\mathrm{T}$ cells. However, when $\mathrm{T}$ cells are exposed to an antigen for a long time, PD-1 expression is upregulated. Furthermore, the antigen-activated $\mathrm{T}$ cells can induce the other cells to overexpress PD-1 by releasing cytokines. PD-L1 inhibits excessive activation of $T$ cells via the PD-1/PD-L1 axis. After PD-L1 binds to PD-1, it leads to phosphorylation of the intracellular domain of $\mathrm{T}$ cell receptors (TCRs), recruiting tyrosine phosphatases SHP-1 and SHP-2. These phosphatases can dephosphorylate several key proteins in the TCR pathway and inhibiting the transcription of related genes and inhibiting $\mathrm{T}$ cell function. Therefore, PD-1/PD-L1 axis play a crucial role in $\mathrm{T}$ cell activation and suppression.

Tumor cells overexpress PD-L1 and continue to activate the PD-1/PD-L1 axis to cause immunosuppression, which promotes tumor-related $\mathrm{T}$ cell apoptosis, transforming $\mathrm{T}$ cells in peripheral and lymphoid tissues into loss-offunction regulatory T cells (Tregs). Exhausted T cells (TEX) inhibit the activation of initial $\mathrm{T}$ cells and the function of effector T cells, and PD-L1 expressed on the surface of immune cells can also affect the response of anti-tumor CD8 + T cells. This is a significant cause of tumor immune evasion. Therefore, lifting the immune brake of PD-1/ PD-L1 is an important strategy for tumor prevention. By releasing the "immune brake" of PD-1 and CTLA-4, Allison and Honjo strengthened the immune system. The excellent results achieved by this method, which represents a milestone in tumor treatment, earned it the 2018 Nobel Prize in Medicine.

At present, PD-1/PD-L1 and CTLA-4 are the main clinical immune checkpoints. PD-1 monoclonal antibodies include pembrolizumab, nivolumab, atezolizumab, avelumab, durvalumab, and cemiplimab-rwlc, and the CTLA-4 monoclonal antibody ipilimumab has been approved by the FDA. They have achieved significant results in many tumors, especially melanoma, non-small cell lung cancer (NSCLC), kidney cancer, urinary tract tumors, squamous cell carcinoma, Merkel cell carcinoma, breast cancer, and colorectal cancer. The idea of checkpoint treatment has far-reaching significance for the clinical treatment of malignant tumors.

\section{Research progress on the relationship between intestinal flora and tumor immunity, tumor formation, and tumor development}

Intestinal flora regulates physiological processes including metabolism, inflammation, and immunity. The intestinal flora is mainly made up of obligate anaerobic bacteria, such as Bacteroides, Eubacterium, Bifidobacterium, and Fusobacterium. Under normal physiological conditions, the intestinal flora interacts with the intestinal epithelium and interstitial cells and plays an important role in maintaining the body's immune balance, digestion, and nutrient absorption. However, the stimulation of certain factors can cause imbalance of the intestinal flora, leading to disease. In recent years, the development of high-throughput sequencing technology has gradually uncovered a close relationship between intestinal flora and immune response and tumor development, as well as its association with the efficacy of various anti-tumor drugs.

Filamentous bacteria in the intestine can stimulate DC and macrophages to secrete IL-23, which can promote Th-17 differentiation and IL-17 production. This has a strong tumor promoting effect in the intestine, especially in NSCLC (1). Viaud et al. tested the composition of fecal flora in breast cancer patients. The results showed that the higher the histological grade of the tumor, the greater the 
number of B. trachoma in the gastrointestinal tract, which suggested that the intestinal flora is also closely related to the degree of tumor malignancy (2). Peuker et al. found that intestinal microbes can combine with toll-like receptors (TLRs) in small intestinal epithelial cells to activate the dendritic cell CaN-NFAT signaling pathway and promote the proliferation of cancer cells (3). Yu et al. described how the combination of lipopolysaccharide, a metabolite of intestinal flora, and hepatocyte TLR4 promoted the occurrence of liver cancer. The consumption of TLR4 can reduce the incidence of liver cancer and inhibit tumor growth. However, TLR4 can also promote the occurrence of lung, pancreas, colon, and skin cancer (4).

Intestinal flora also has an influence on the effectiveness of tumor treatments. Oxaliplatin is a commonly used clinical chemotherapeutic drug. Iida et al. found that probiotic bacteria such as L. acidophilus can stimulate immune cells to secrete ROS, which is consistent with the mechanism of oxaliplatin in killing tumor cells (5). Viaud et al. found that after applying cyclophosphamide to tumorbearing mice, the amount of Gram-positive bacteria, such as Lactobacillus johnsonii, Lactobacillus murine, and enterococcus hirae, were increased. They also observed that the helper Th-17 cell response and the anti-tumor effect of cyclophosphamide were reduced in sterile mice treated with antibiotics without Gram-positive bacteria (2). As described below, a relationship also exists between intestinal flora and the effect of tumor immunotherapy.

\section{The current status of immune checkpoints in cancer treatment}

ICIs are currently used to treat melanoma, NSCLC, liver cancer, gastric cancer, renal cell cancer, and other cancers.

\section{Melanoma}

In a phase II clinical trial of Nivolumab (PD-1 inhibitor) combined with Ipilimumab (CTLA4 inhibitor) and nivolumab alone, $16 / 35$ patients (46\%, $95 \%$ CI: $29-$ $63 \%$ ) who received the combination therapy showed an intracranial response. Meanwhile, of those treated with Nivolumab alone, 5/25 (20\%, 95\% CI: 7-41\%) patients experienced intracranial treatment response. This shows that combined treatment can achieve a higher objective response rate (ORR), For these patients, the combination therapy should be considered as the first-line treatment (6). Another clinical phase III study that compared the safety and efficacy of Nivolumab and Ipilimumab combination therapy with Nivolumab or Ipilimumab alone in patients with stage III or IV melanoma showed that the median progression-free survival (PFS) rates were 11.5, 6.9, and 2.9 months in the combined treatment, nivolumab alone, and ipilimumab alone groups, respectively, which indicates that the combined use of ICIs has a stronger therapeutic effect on melanoma (7).

\section{NSCLC}

A phase I clinical trial of Nivolumab combined with IL-15 superagonist ALT-803 to treat NSCLC saw 21/23 patients complete 4 dose levels $(6,10,15$, and $20 \mu \mathrm{g} / \mathrm{kg}$ ) of ALT-803 combined with Nivolumab $(3 \mathrm{mg} / \mathrm{kg})$. The enrolled patients experienced no serious side effects, which suggested that the method of ALT-803 combined with Nivolumab is safe; however, its clinical efficacy still needs further evaluation in more studies (8). In another study, Nivolumab was used to treat 38 patients with progressive NSCLC. It was found that the ORR of these patients was $23.7 \%$, and the median PFS was 91 days. Immune-related side effects were observed in 14 patients. It is worth noting that the patients who experienced immune-related side effects had a higher ORR $(\mathrm{P}<0.001)$ and longer PFS $(\mathrm{P}<0.001)$, suggesting that the immune-related side effects is related to the efficacy of Nivolumab; this needs to be verified by a large sample study (9).

\section{Digestive system tumors}

The American Society of Clinical Oncology Congress reported on a multicenter, open phase IB clinical study of Pembrolizumab (a PD-1 inhibitor) (10), which included 162 patients with adenocarcinoma of the stomach or gastroesophageal junction. PD-L1 positive patients were treated with Pembrolizumab ( $10 \mathrm{mg} / \mathrm{kg}$ once every 2 weeks) for 24 months. Treatment was discontinued when the patient reached complete remission, the disease progressed, or an intolerable adverse reaction occurred. The results found that the ORR in this group of patients was $22.2 \%$, the median PFS was 1.9 months, and the median overall survival (OS) time was 11.4 months. Furthermore, the study showed PD-1 expression to be correlated with OS, suggesting that positive expression of PD-L1 can be used as a clinical screening indicator. Another study included 259 patients with gastric or gastroesophageal junction adenocarcinoma. Through Pembrolizumab targeted therapy, it was found that the ORR of the PD-L1 positive group (15.5\%) was significantly higher than that of the PD-L1 negative group (6.4\%). However, the complete remission rate of the PD$\mathrm{L} 1$ positive group $(2.0 \%)$ was lower than that of the $\mathrm{PD}-\mathrm{L} 1$ 
negative group (2.8\%). The above studies confirmed that Pembrolizumab can fight the occurrence and development of gastric cancer through targeting of PD-1 (11).

Tremelimumab (CTLA-4) was approved by the FDA for clinical treatment. In the phase II clinical trial (NCT01008358), Tremelimumab was used to treat 17 patients with advanced liver cancer accompanied by chronic hepatitis $\mathrm{C}$ virus infection. The ORR was $17.6 \%$, the disease control rate (DCR) was $76.4 \%$, the time to progression (TTP) was 6.5 months, and a significant decrease in viral load was also observed. In another clinical trial (NCT01853618), Tremelimumab combined with arterial chemoembolization, radiofrequency ablation, and cryoablation achieved useful clinical effects in 32 patients with advanced liver cancer and 9 patients with cholangiocarcinoma. The median PFS of the patients was 5.7 months (12).

A phase II trial evaluated the efficacy of Ipilimumab in advanced pancreatic cancer, and the results showed that none of the 27 subjects achieved a tumor response that met RECIST criteria (11). A phase Ib clinical trial (NCT01473940) evaluated the efficacy of Ipilimumab and gemcitabine in the treatment of stage III-IV or inoperable recurrent pancreatic cancer. Of the 16 patients enrolled, 2 had partial remission (PR) and 5 had stable disease (SD). The median PFS and OS rates were 2.5 and 8.5 months, respectively. Ipilimumab can also be used in combination with pancreatic cancer vaccines. A phase Ib single-arm trial (NCT00836407) compared the efficacy between ipilimumab or GM-CSF gene-transduced tumor vaccine (GVAX) for treating pancreatic cancer patients who had previously received combination treatment. Two and three patients achieved SD in the single-agent group and the combined treatment group, respectively. The combined treatment group had better survival and prognosis. The median OS rate was $5.7 v s .3 .6$ months, the 1-year OS rate was $27 \% v$ s. $7 \%$. In general, Ipilimumab combined with chemotherapy or a vaccine is more effective than monotherapy in treating pancreatic cancer.

\section{Other tumors}

Motzer et al. (13) compared the clinical effect of the combination of Nivolumab and Ipilimumab with that of Sunitinib (tyrosine kinase inhibitor) alone in the treatment of advanced renal cell carcinoma (RCC). The results showed that compared to Sunitinib alone $(60 \%, 95 \%$ CI: 55-65\%), the Nivolumab and Ipilimumab combined immunotherapy group had a higher OS rate $(75 \%, 95 \%$
CI: $70-78 \%$ ) and ORR (42\% vs. $27 \%, \mathrm{P}<0.001)$, and $9 \%$ of patients achieved complete remission. Compared to Sunitinib alone, Nivolumab and Ipilimumab combined immunotherapy can reduce the risk of advanced RCC, reduce disease-related symptoms, and improve patients' quality of life. In the clinical phase I trial of Falchook et al. (14), Cemiplimab (PD-1 monoclonal antibody) had a lasting therapeutic effect on progressive skin squamous cell carcinoma, and, more than 16 months after treatment, the patients had experienced no disease recurrence. The results of phase II clinical trials conducted by Migden et al. (15) showed that the ORR of Cemiplimab in the treatment of progressive skin squamous cell carcinoma was $47 \%$. The effect of checkpoint suppression on skin tumors was confirmed.

\section{Current status of the impact of the application of checkpoint inbibitors and the interaction of intestinal flora in tumor treatment}

There is currently no consensus regarding for the influence intestinal flora on the effect of ICI therapy; however, studies have generally found that the two are linked (Table 1). One study used 16S ribosomal RNA sequencing (16S ribosomal RNA sequencing), metagenomics shotgun sequencing (MSS), and quantitative PCR (quantitative polymerase chain reaction, $\mathrm{qPCR}$ ) technology to sequence the intestinal microflora of patients before ICI treatment. It was found that beneficial microbiota was related to the effect of ICI therapy. Dubin et al. (22) sequenced the bacterial flora of 34 melanoma patients and found that those with melanoma rich in Bacteroidetes had a lower incidence of colitis after the application of the CTLA-4 blocker ipilimumab. Chaput et al. (16) used 16S ribosomal RNA sequencing to divide 26 melanoma patients into group A (Bacteroides) and group B (Faecalibacterium genus, Firmicutes) according to the difference in bacterial enrichment and applying the CTLA-4 treatment to two groups of patients. The inhibitor Ipilimumab Patients with Faecalibacterium, accompanied by a higher incidence of colitis, increased peripheral blood ICOS $+\mathrm{T}$ cells and sCD25, and decreased Treg cells, were found to have better PFS and OS than patients rich in Bacteroides, Frankel et al. (23) applied anti-CTLA-4 and PD-1 ICIs (ipilimumab, nivolumab, and pembrolizumab) in a prospective study of melanoma patients. The patients were given ipilimumab and nivolumab (IN) in combination, or pembrolizumab single-agent use (P). MSS was carried out before treatment. 
Table 1 Bacteria associated with ICTs treatment in humans

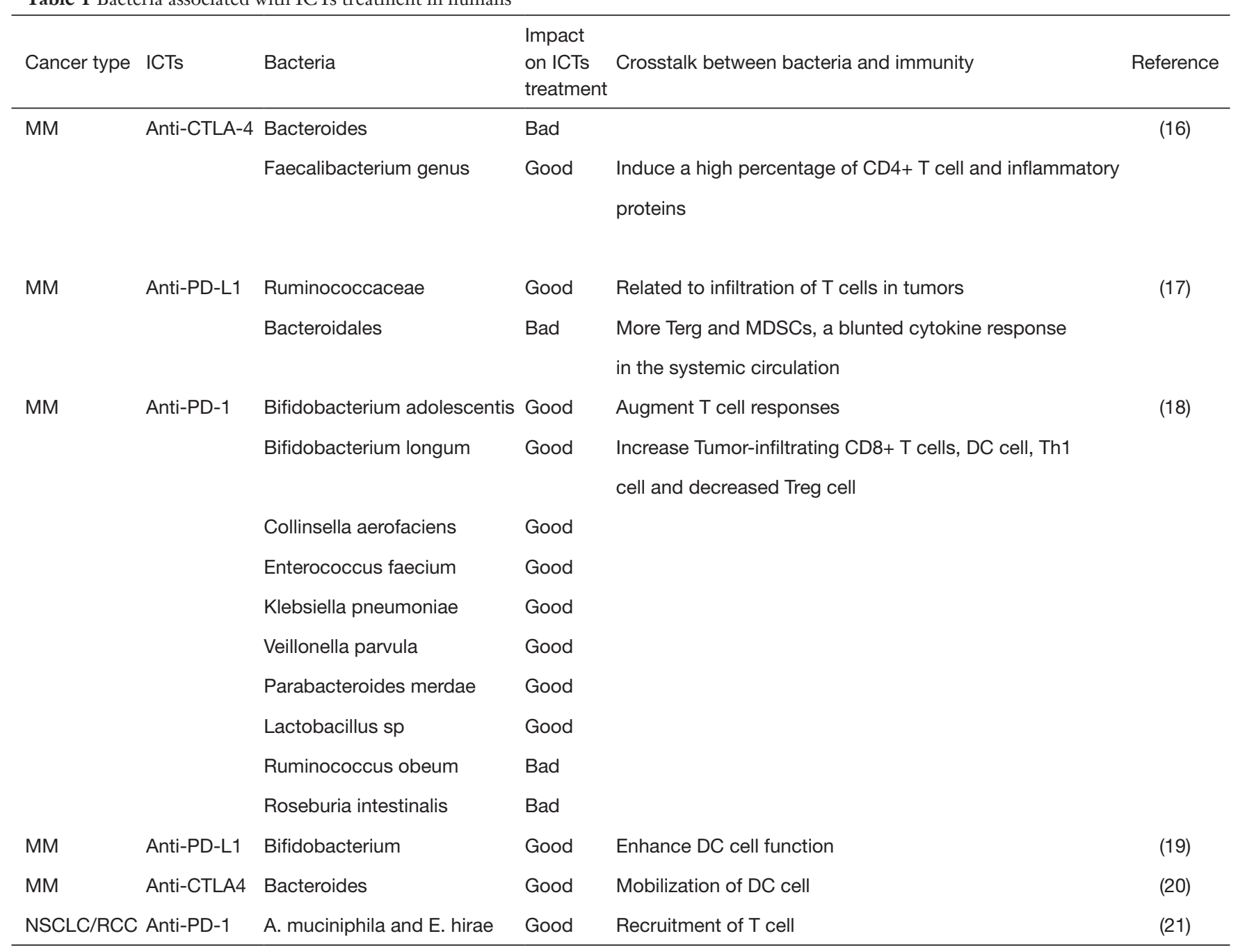

ICT, immnue checkpoint target; MM, metastatic melanoma.

IN and $\mathrm{P}$ produced response rates of $67 \%$ and $23 \%$, respectively, and all ICI responders were rich in Bacteroides caccae. In the responsive patients in the IN group, the intestinal flora was rich in Faecalibacterium prausnitzii, Bacteroides thetaiotaomicron, Holdemania filiformis. In the responsive patients in the $\mathrm{P}$ group, the intestinal flora was rich in Dorea formicigenerans. Unbiased metabolomic profiling (Unbiased Metabolomic Profiling) analysis of all responsive patients showed high levels of erucic acid. The flora concentrated in the responsive patients was Faecalibacterium prausnitzii, Bacteroides thetaiotaomicron, Holdemania filiformis, and Bacteroides caccae. These patients also had a high expression of intestinal bacterial enzymes related to fatty acid synthesis and inositol phosphate metabolism. Gopalakrishnan et al. (17) studied 43 cases of melanoma patients treated with anti-PD-1 antibodies, and the intestinal flora of the responsive patients was enriched in Faecalibacterium prausnitzii, Ruminococcus bromii, Porphyromonas pasteri, Clostridium hungatei, and Phascolarctobacterium faecium. Immunohistochemistry (IHC) and blood flow cytometry showed that the levels of CD8 + T cells and CD68 + HLA-Dr + CD163 + myeloid DC cells were increased, whereas the levels of Tregs and myeloid inhibitory cells were decreased. All of the responsive patients had increased amino acid synthesis. Matson et al. (18) treated 42 melanoma patients with PD-1 antibody (38 cases) and anti-CTLA4 antibody. The analysis showed that Enterococcus faecium, Collinsella aerofaciens, 
Bifidobacterium adolescentis, Klebsiella pneumoniae, Veillonella parvula, Parabacteroides merdae, Lactobacillus sp, and Bifidobacterium longum were in higher abundance in the intestinal tracts of the responders. Furthermore, PCR technology detected high levels of PD-1 and PD-L1 mRNA expression, and the level of CD8 + T cell infiltration was also high.

Routy et al. (21) used the MSS method to sequence and blast analyze fecal samples from 60 patients with NSCLC and 40 patients with RCC. Response to AntiPD-1 Immunotherapy, Akkermansia muciniphila, Lachnospiraceae, Erysipelotrichaceae bacterium 5-2-64, Enterococcus faecium, Alistipes indistinctus, Bacteroides caccae, Bacteroides xylanisolvens, and Bacteroides nordii were more enriched.

Intestinal floras on the therapeutic effect of ICIs are dominant, in fact, some experts also studied the immunotherapy effect of oral flora on ICIs, but did not get meaningful results. Gopalakrishnan et al. have proved that oral flora has no effect on the efficacy of ICIs various research methods. Through 16S RNA sequencing, the oral flora and intestinal flora are diverse, a high abundance of bacteria of the order Lactobacillales in the oral microbiome and Bacteroidales in the fecal microbiome. Bipartite network analysis demonstrated both communities are distinct in terms of their compositional structure. When tested the relationship of diversity and progression-free survival (PFS) in cohort by stratifying patients based on tertiles of inverse Simpson scores, No differences in PFS were noted when comparing diversity of the oral microbiome. they built a phylogenetic tree based on sequence alignment data and divide all identified OTUs into related OTU (crOTU) clusters. This technique involves comparing the abundance of different potential bacterial species based on $16 \mathrm{~S}$ sequence similarity and helps to solve the sparse distribution of OTU abundance observed in the absence of this method, and analysis of crOTU in the oral microbiome showed no significant relationship with treatment response.

\section{Animal experiment research on the relationship between ICI therapy and intestinal flora}

In recent years, animal experiments have offered proof that the intestinal flora is related to the efficacy of ICIs. Sivan et al. (19) compared the growth of melanoma in mice with different symbiotic flora and observed differences in antitumor immunity, and these immune differences could be eliminated by fecal transplantation or cohabitation.
It was confirmed by $16 \mathrm{~S}$ ribosomal RNA sequencing that Bifidobacterium is beneficial for PD-L1 inhibitors in treating tumors, and the enhanced DC cell function and CD8 (+) T cells mediate the anti-tumor mechanism. Vétizou et al. (20) found that MCA205 sarcoma mice had tumor shrinkage after CTLA-4 was blocked, while tumorbearing mice treated with sterile mice or antibiotics did not respond to CTLA-4 blockade. Bacteroides fragilis, Bacteroides fragilis, and Burkholderia are beneficial in blocking the CTLA-4 immune response. Intratumoral CD11b+ DCs secrete IL-12, and infiltrating T cells are associated with a good therapeutic effect. In the study of Tanoue et al. (24), MC38 tumor-bearing mice showed a good response to PD-1/CTLA-4 blockers, but germfree mice or pretreatment with antibiotics, the response weakened. The former stimulates CD8 $+\mathrm{T}$ cells to produce IFN- $\gamma$. After the infusion of 11 bacterial types into tumorbearing mice via intragastric administration, not only was the response of PD-1/CTLA-4 blockade restored, but the ability to resist Listeria infection was improved.

Some scholars obtained feces from patients with good ICI response and transplanted them into ICI-treated tumor-bearing mice. A greater effect was greater, and there was increased infiltration of tumor CD8 $+\mathrm{T}$ cells and myeloid-derived DC cells. The stools of melanoma patients (responders/non-responders) were administered via intragastric administration to tumor-bearing mice treated with anti-PD-L1. After one month of observation, the tumors of the mice after the administration of patients feces of PD-L1 responders were significantly reduced, and the levels of CD8 + T cells and myeloid CD $45+\mathrm{CD} 11 \mathrm{~b}$ + Ly6G + DC cells were increased. Meanwhile, the levels of splenic myeloid CD11b + CD11c + suppressor cells and Fox $3 \mathrm{P}+\mathrm{CD} 4+$ Tregs were decreased, and Faecalibacterium prausnitzii was increased significantly in the mouse feces (17). Matson et al. administered the feces of responders/nonresponders to sterile mice via intragastric administration. After inoculating the mice with B16SIY melanoma cells and administering anti-PD-L1 treatment, they observed that the tumors of the mice exposed to the feces of the responsive patients had grown at a slower rate and these mice had higher levels of Spleen IFN $\gamma+\mathrm{CD} 8+\mathrm{T}$ cells and Batf3 + DCs (18). Routy et al. (21) also obtained similar results. The feces of patients with good response to transplantation can promote tumor suppression of checkpoint inhibitors to a certain extent. When Akkermansia muciniphila was used instead of feces to perfuse tumor-bearing mice, growth was inhibited in $40 \%$ of the tumors, and tumor cell CCR9 + 


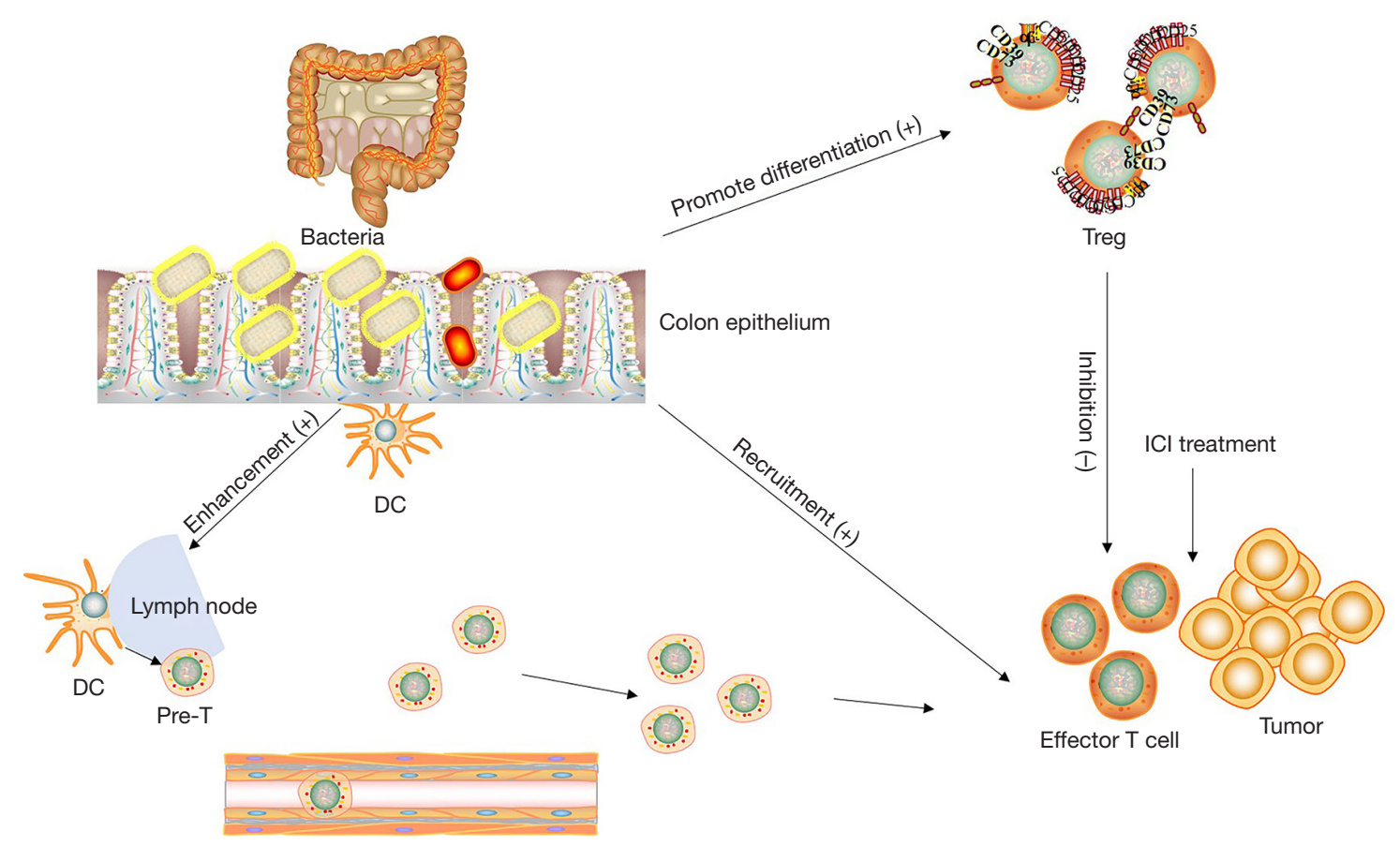

Figure 1 In the treatment of ICIs, intestinal flora played a positive role in the presentation of tumor antigens, T cell transport, activation, recruitment and tumor infiltration. Also. Some types of bacteria could mobilize to activate Treg cells and suppress tumor immunity. DC, dendritic cell.

CD4 + T cell infiltration was increased, which is related to IL-12, or IL-12 dependent.

Although many studies have confirmed that a relationship exists between intestinal flora and response to ICI therapy, the exact mechanism is still unclear. The intestinal flora antigen stimulating the model pattern recognition receptor (PRR) of DC cells and activating the innate immune response may be one potential mechanism. Others include the intestinal flora antigens mimicking neoplastic antigens and playing the role of tumor vaccines, and intestinal flora producing small molecules to activate CTL function.

\section{Potential interactions in intestinal flora and ICIs}

According to the existing research results of intestinal flora and ICIs, multiple steps of the cancer-immunity cycle are promoted or inhibited by intestinal flora (Figure 1). The first step is the presentation of tumor antigens, the antigen presentation function of DC cells is very powerful, Vétizou $M$ found Bacteroides could mobilize and activate DC cells in MM patients treated with ICIs. Sivan and Matson found in MM patients treated with ICIs, Bifidobacterium can increase the number of DC cells. The second step is the process of $\mathrm{T}$ cell transport and infiltration into tumor tissue, Routy B observrd the phenomenon of $\mathrm{T}$ cell recruitment in tumor tissue in well-respond NSCLC and RCC patients with A. muciniphila and E. hirae enrichment. Matson and further found Bifidobacterium longum could increase Tumor-infiltrating CD8+ T cells. Gopalakrishnan found Ruminococcaceae was related to the infiltration of $\mathrm{T}$ cells in tumors. Faecalibacterium genus induced a high percentage of CD4+ T cell and inflammatory proteins. In addition, Faecalibacterium prausnitzii and Clostridia may promote Treg differentiation and increase the Treg number. Bacteroidales cause more Terg amd myeloidderived suppressor cells (MDSCs), a blunted cytokine responsein in the systemic circulation, which in turn causes immunosuppression.

\section{Comprehensive analysis of various studies}

The intestinal flora plays a dual role in tumors. First, it can promote the occurrence and development of tumors, and second, it also affects the efficacy and adverse reactions of tumor treatment. Various studies have involved a wide variety of bacteria related to the efficacy and adverse 
reactions of immunotherapy, including Akkermansia, Bifidobacterium, and Fecal bacteria. These studies are consistent in some respects. If the thick-walled phylum is positively correlated with good treatment results (Ackerman, Lactobacillus, Ruminococcus, and Fecal bacterium belong to the thick-walled phylum), the upstream substrates and downstream pathways of different bacteria should be in common. For example, Routy emphasized the role of Ackerman, while Gopalakrishnan and Chaput found that the genus Faecalis is more abundant. Therefore, different types of tumors have different beneficial bacterial groups. Moreover, there may be multiple beneficial bacterial groups in one tumor, and one bacterial group may also be beneficial bacteria in multiple tumors. Further research is needed to establish the beneficial bacteria in different tumor types. A combination of good bacteria can maximize the treatment effect for patients.

\section{Outlook}

The effect of intestinal flora on the therapeutic effect of immune checkpoint inhibitors is now determined, and it is very promising in the future prevention and treatment of cancer. Firstly, the composition of the gut microbiota can potentially be a predictive biomarker and treatment target in ICIs therapy. Secondly, fecal microbiota transplantation (FMT) is considered a tremendous method to enhance the efficacy of ICIs and overcome drug resistance. Thirdly, multiple tumor immunotherapy centers should conduct long-term cooperation to unify the pre-test bacterial detection methods and treatment methods in the test and the corresponding biochemical detection technology. In addition, some scholars have confirmed that the microbiome obtained from feces is not a perfect substitute for the intestinal mucosa-associated microbiome, so can it improve the technology and more accurately understand the actual composition of the intestinal microflora? Moreover, we can start the research from the generally recognized beneficial bacteria, which is economical and potentially successful. Lastly, the composition of intestinal flora of different races is different, and even there are differences between individuals. If we can build a sequencing database of intestinal flora and immunotherapy, we can achieve accurate individualized treatment plans.

\section{Summary}

According to the current research, intestinal flora has an impact on the tumor treatment of ICIs; however, the research in this area is still inadequate.

First, although many medical centers have conducted studies on the relationship between ICI efficacy and intestinal flora, they generally include a small number of patients, a single tumor type, a unique patient group, differences in stool collection techniques and bacterial sequencing methods, and differences in follow-up time, Drug effects and so on, have led to a variety of results and limited the accuracy of the comparisons. According to the aforementioned differences in the beneficial flora of different tumor responders, it is indicated that each type of cancer has specific immune gut flora. To this end, the sample size should be increased and the scope of tumor research should be expanded and should not focus on the tumors mentioned above. Multicenter cooperative research, unified patient management modes, sample processing procedures, and biological information analysis methods will help to address this problem.

Second, the studies to date did not consider other factors that may affect the composition of the intestinal flora, such as the patient's race, age, delivery method, feeding method, long-term lifestyle habits, eating habits, drugs (antibiotics, etc.), chronic diseases, and other factors. Intestinal flora composition is greatly influenced by environmental factors, and genetic factors only account for $2 \%$ to $8 \%$ of the observed variations. Differences between the included patients are likely to produce heterogeneity in the results. In addition, studies have shown antibiotics can regulate the intestinal flora and adversely affect the response to ICIs. Derosa et al. (25) found that PD-1/PD-L1 had a greater effect in patients who did not receive antibiotics than in those who did in NSCLC and kidney cancer. Cancer treatment is more effective in patients who do not use antibiotics, and they also have significantly better median OS and PFS rates than those who do. However, infection is common in cancer patients, and so the use of antibiotics during ICI treatment needs comprehensive evaluation and an expert consensus needs to be reached. In addition to antibiotics, proton pump inhibitors (26), metformin (27), and glucocorticoids (28) also affect the intestinal flora.

Furthermore, in ICI-related animal experiments and clinical studies, the methods used to change the intestinal flora-fecal transplantation and antibiotic intervention (animal) and oral probiotics (human)—are simpler than the body's normal processes compared with the actual intestine. There is a definite difference in the plasticity of the tract flora, so whether it involves the remodeling of human 
intestinal flora or an animal model, the construction needs to be improved.

Trials also involve ethical issues, such as whether changes in the intestinal flora will cause new diseases to develop in the body. For patients with cancer, fecal transplantation also carries the risk of infection and may have an impact on the patient's psychology. Further consideration and research into these issues is called for.

\section{Acknowledgments}

Funding: Supported by the Natural Science foundation of China (grant 81872101).

\section{Footnote}

Reporting Checklist: The authors have completed the Narrative Review reporting checklist. Available at http:// dx.doi.org/10.21037/atm-20-4535

Conflicts of Interest: All authors have completed the ICMJE uniform disclosure form (available at http://dx.doi. org/10.21037/atm-20-4535). The authors have no conflicts of interest to declare.

Ethical Statement: The authors are accountable for all aspects of the work in ensuring that questions related to the accuracy or integrity of any part of the work are appropriately investigated and resolved.

Open Access Statement: This is an Open Access article distributed in accordance with the Creative Commons Attribution-NonCommercial-NoDerivs 4.0 International License (CC BY-NC-ND 4.0), which permits the noncommercial replication and distribution of the article with the strict proviso that no changes or edits are made and the original work is properly cited (including links to both the formal publication through the relevant DOI and the license). See: https://creativecommons.org/licenses/by-nc-nd/4.0/.

\section{References}

1. Song Y, Yang JM. Role of interleukin (IL)-17 and T-helper (Th) 17 cells in cancer. Biochem Biophys Res Commun 2017;493:1-8.

2. Viaud S, Saccheri F, Mignot G, et al. The intestinal microbiota modulates the anticancer immune effects of cyclophosphamide. Science 2013;342:971-6.
3. Peuker K, Muff S, Wang J, et al. Epithelial calcineurin controls microbiota-dependent intestinal tumor development. Nat Med 2016;22:506-15.

4. Yu LX, Schwabe RF. The gut microbiome and liver cancer: mechanisms and clinical translation. Nat Rev Gastroenterol Hepatol 2017;14:527-39.

5. Iida N, Dzutsev A, Stewart CA, et al. Commensal bacteria control cancer response to therapy by modulating the tumor microenvironment. Science 2013;342:967-70.

6. Long GV, Atkinson V, Lo S, et al. Combination nivolumab and ipilimumab or nivolumab alone in melanoma brain metastases: a multicentre randomised phase 2 study. Lancet Oncol 2018;19:672-81.

7. Hodi FS, Chiarion-Sileni V, Gonzalez R, et al. Nivolumab plus ipilimumab or nivolumab alone versus ipilimumab alone in advanced melanoma (CheckMate 067): 4-year outcomes of a multicentre, randomised, phase 3 trial. Lancet Oncol 2018;19:1480-92.

8. Wrangle JM, Velcheti V, Patel MR, et al. ALT-803, an IL-15 superagonist, in combination with nivolumab in patients with metastatic non-small cell lung cancer: a nonrandomised, open-label, phase $1 \mathrm{~b}$ trial. Lancet Oncol 2018;19:694-704.

9. Sato K, Akamatsu H, Murakami E, et al. Correlation between immune-related adverse events and efficacy in non-small cell lung cancer treated with nivolumab. Lung Cancer 2018;115:71-4.

10. Muro K, Chung HC, Shankaran V, et al. Pembrolizumab for patients with PD-L1-positive advanced gastric cancer (KEYNOTE-012): a multicentre, open-label, phase 1b trial. Lancet Oncol 2016;17:717-26.

11. Fuchs CS, Doi T, Jang RW, et al. Safety and Efficacy of Pembrolizumab Monotherapy in Patients With Previously Treated Advanced Gastric and Gastroesophageal Junction Cancer: Phase 2 Clinical KEYNOTE-059 Trial. JAMA Oncol 2018;4:e180013.

12. Le DT, Lutz E, Uram JN, et al. Evaluation of ipilimumab in combination with allogeneic pancreatic tumor cells transfected with a GM-CSF gene in previously treated pancreatic cancer. J Immunother 2013;36:382-9.

13. Motzer RJ, Tannir NM, McDermott DF, et al. Nivolumab plus Ipilimumab versus Sunitinib in Advanced Renal-Cell Carcinoma. N Engl J Med 2018;378:1277-90.

14. Falchook GS, Leidner R, Stankevich E, et al. Responses of metastatic basal cell and cutaneous squamous cell carcinomas to anti-PD1 monoclonal antibody REGN2810. J Immunother Cancer 2016;4:70.

15. Migden MR, Rischin D, Schmults CD, et al. PD-1 Blockade 
with Cemiplimab in Advanced Cutaneous Squamous-Cell Carcinoma. N Engl J Med 2018;379:341-51.

16. Chaput N, Lepage P, Coutzac C, et al. Baseline gut microbiota predicts clinical response and colitis in metastatic melanoma patients treated with ipilimumab. Ann Oncol 2019;30:2012.

17. Gopalakrishnan V, Spencer CN, Nezi L, et al. Gut microbiome modulates response to anti-PD-1 immunotherapy in melanoma patients. Science 2018;359:97-103.

18. Matson V, Fessler J, Bao R, et al. The commensal microbiome is associated with anti-PD-1 efficacy in metastatic melanoma patients. Science 2018;359:104-8.

19. Sivan A, Corrales L, Hubert N, et al. Commensal Bifidobacterium promotes antitumor immunity and facilitates anti-PD-L1 efficacy. Science 2015;350:1084-9.

20. Vétizou M, Pitt JM, Daillère R, et al. Anticancer immunotherapy by CTLA-4 blockade relies on the gut microbiota. Science 2015;350:1079-84.

21. Routy B, Le Chatelier E, Derosa L, et al. Gut microbiome influences efficacy of PD-1-based immunotherapy against epithelial tumors. Science 2018;359:91-7.

22. Dubin K, Callahan MK, Ren B, et al. Intestinal microbiome analyses identify melanoma patients at risk for checkpoint-blockade-induced colitis. Nat Commun

Cite this article as: Wang D, Hao H, Li X, Wang Z. The effect of intestinal flora on immune checkpoint inhibitors in tumor treatment: a narrative review. Ann Transl Med 2020;8(17):1097. doi: 10.21037/atm-20-4535
2016;7:10391.

23. Frankel AE, Coughlin LA, Kim J, et al. Metagenomic Shotgun Sequencing and Unbiased Metabolomic Profiling Identify Specific Human Gut Microbiota and Metabolites Associated with Immune Checkpoint Therapy Efficacy in Melanoma Patients. Neoplasia 2017;19:848-55.

24. Tanoue T, Morita S, Plichta DR, et al. A defined commensal consortium elicits CD8 T cells and anti-cancer immunity. Nature 2019;565:600-5.

25. Derosa L, Hellmann MD, Spaziano M, et al. Negative association of antibiotics on clinical activity of immune checkpoint inhibitors in patients with advanced renal cell and non-small-cell lung cancer. Ann Oncol 2018;29:1437-44.

26. Imhann F, Bonder MJ, Vich Vila A, et al. Proton pump inhibitors affect the gut microbiome. Gut 2016;65:740-8.

27. Forslund K, Hildebrand F, Nielsen T, et al. Corrigendum: Disentangling type 2 diabetes and metformin treatment signatures in the human gut microbiota. Nature 2017;545:116.

28. Maher VE, Fernandes LL, Weinstock C, et al. Analysis of the Association Between Adverse Events and Outcome in Patients Receiving a Programmed Death Protein 1 or Programmed Death Ligand 1 Antibody. J Clin Oncol 2019;37:2730-7. 